\title{
Solar surveillance with CLIMSO: instrumentation, database and on-going developments
}

\author{
Frédéric Pitout $^{1, *}$, Laurent Koechlin ${ }^{1}$, Arturo López Ariste ${ }^{1}$, Luc Dettwiller ${ }^{2}$, and Jean-Michel Glorian ${ }^{1}$ \\ ${ }^{1}$ IRAP, Université Toulouse 3, CNRS, CNES, 9 Avenue du Colonel Roche, BP 44346, 31028 Toulouse Cedex 4, France \\ ${ }^{2}$ Higher Education Section of Lycée Blaise Pascal, 36 Avenue Carnot, 63037 Clermont-Ferrand Cedex, France
}

Received 1 May 2020 / Accepted 27 July 2020

\begin{abstract}
CLIMSO is a suite of solar telescopes installed at Pic du Midi observatory in the southwest of France. It consists of two refractors that image the full solar disk in $\mathrm{H} \alpha$ and CaII $\mathrm{K}$, and two coronagraphs that capture the prominences and ejections of chromospheric matter in $\mathrm{H} \alpha$ and HeI. Synoptic observations are carried out since 2007 and they follow those of previous instruments. CLIMSO, together with its predecessors, offer a temporal coverage of several solar cycles. With a direct access to its images, CLIMSO contributes to real time monitoring of the Sun. For that matter, the national research council for astrophysics (CNRS/INSU) has labelled CLIMSO as a national observation service for "surveillance of the Sun and the terrestrial space environment". Products, under the form of images, movies or data files, are available via the CLIMSO DataBase. In this paper, we present the current instrumental configuration; we detail the available products and show how to access them; we mention some possible applications for solar and space weather; and finally, we evoke developments underway, both numerical to valorise our data, and instrumental to offer more and better capabilities.
\end{abstract}

Keywords: Sun / observation / telescopes / coronagraphs / database

\section{Introduction}

\subsection{A brief history of solar observations at Pic du Midi}

The Pic du Midi observatory is located on top of the Pic du Midi de Bigorre Mountain (latitude $42^{\circ} 56^{\prime} 11^{\prime \prime} \mathrm{N}$; longitude $0^{\circ} 08^{\prime} 34^{\prime \prime} \mathrm{E}$; altitude $2876 \mathrm{~m}$ ), near the cities of Bagnères-deBigorre and Tarbes in the south-west of France. It was inaugurated in 1882 after 4 years of construction in harsh conditions. It was initially a meteorological station and astronomical observations came a little later. In 1892, the new director Émile Marchand, who was interested in Sun-Earth connections (e.g., Marchand, 1904), set up the first routine solar observations.

In the early 1930s, Bernard Lyot invented the coronagraph, an ingenious instrument that allows creating artificial solar eclipses and observing the solar prominences and corona (Lyot, 1930). He conducted many tests and observations at the Pic du Midi observatory (Lyot, 1930, 1935), where there has been a long tradition of solar observation ever since. Indeed, several instruments taking over the preceding one have almost continuously observed our star for 90 years (Demarcq et al., 1965; Noëns \& Wurmser, 2000; Meunier et al., 2007; Romeuf

\footnotetext{
*Corresponding author: frederic.pitout@irap.omp.fr
}

et al., 2007). Although the instruments were different in sizes and performances, the resulting data set is quite valuable for long-term studies, like solar activity over the cycles (4 full magnetic cycles are covered).

In the late 1990s, the Pic du Midi faced a severe budget situation and only two options were envisaged: closing down the whole observatory and ceasing all astronomical observations, or opening a part of the observatory to the public and searching external funding to maintain some of the scientific activities. The latter option was chosen. As the solar observations are concerned, a non-profit association of amateur astronomers was founded, the "observateurs associés" (OA), literally "associated observers" in French. The OA, thanks to a private sponsor, manage to purchase several solar instruments; CLIMSO (Christian Latouche IMageurs SOlaires) was born.

In 2007, the French national research council for astronomy and astrophysics (CNRS/INSU) labelled the CLIMSO instruments as a national observation service for solar surveillance (SNO-6). This label, on top of the recognition it gives the project, allows the employment of dedicated staff and the allocation of funding at the national level (CNRS/INSU) or at the regional level (Toulouse University, Midi-Pyrénées observatory). In 2020, the CLIMSO database (CLIMSO-DB) got the SNO-5 label for data archiving and dissemination. 
Table 1. Overview of CLIMSO instrumentation and products.

\begin{tabular}{|c|c|c|c|c|c|}
\hline & L1 & L2 & $\mathrm{C} 1$ & \multicolumn{2}{|c|}{$\mathrm{C} 2$} \\
\hline Effective focal length (mm) & 1350 & 1250 & 1244 & \multicolumn{2}{|c|}{1133} \\
\hline Filter $\lambda(\mathrm{nm})$ & 656.28 & 393.37 & 656.28 & 1083.0 & 1074.7 \\
\hline Filter $\Delta \lambda(\mathrm{nm})$ & 0.05 & 0.25 & 0.25 & 0.25 & 0.25 \\
\hline Resolution (arcsec/pixel) & 1.2 & 1.2 & 1.2 & 1.2 & 1.2 \\
\hline Exposure time (s) & 0.04 & 0.05 & 0.001 & $0.1(\times 20)$ & $0.6(\times 10)$ \\
\hline Image formats & & & FITS,. & & \\
\hline Movie format & & & MP & & \\
\hline
\end{tabular}

\subsection{Solar surveillance for space weather}

Nowadays, space weather predictions rely on numerous observational and modelling efforts. On the observational side, real-time imaging of the Sun in various wavelengths is needed to spot and watch active regions and filaments that could be ejected. Then models propagate the solar wind and extreme events such as coronal mass ejections (CME), corotating interaction regions (CIR) or solar energetic particles (i.e. Propagation Tool; Rouillard et al., 2017). Measurements in the solar wind are also necessary to have a precise idea of the properties of the solar wind and the interplanetary magnetic field (IMF) that will eventually hit the Earth. The last step is the modelling and forecast of the expected effects of the solar wind disturbances in the geospace, which includes the magnetosheath, the magnetosphere and the upper neutral and ionised atmosphere.

Until a recent past, our space weather predictions relied entirely on data from solar wind monitors such as the Advanced Composition Explorer (ACE, Stone et al., 1998) or the Deep Space Climate Observatory (DSCOVR; Burt \& Smith, 2012), which provide us with the essential parameters: IMF amplitude and orientation, solar wind density and velocity. These spacecraft are located around the L1 Lagrange point, very close to the Earth, so they do not leave us much time to react. To improve our modelling and reaction time to a possible extreme solar event, we must move back in time and space the observations that feed our models. Space weather predictions should thus rely on solar observations.

To this end, integrated numerical codes, such as the WangSheeley-Arge-Enlil (WSA-ENLIL; Odstrcil \& Pizzo, 1999; Arge \& Pizzo, 2000) or the EUropean Heliospheric FORecasting Information Asset (EUHFORIA; Pomoell \& Poedts, 2018), aim at model the whole chain of physical processes from the surface of the Sun to the Earth. As inputs, those models need basic parameters such as the time of eruptions, the velocity and density of the ejected chromospheric plasma, and the magnetic field at the footprint of the structures and/or within the ejected plasma.

CLIMSO is a system that can play its part in those observational efforts. CLIMSO has contributed to the on-going solar survey at Pic du Midi observatory (Koechlin et al., 2019) and now, we aim at monitoring in real time the full solar disk and near environment, and thus at anticipating possible solar flares and coronal mass ejections. CLIMSO is listed as an asset by the Space Situation Awareness (SSA) service of the European Space Agency (http://swe.ssa.esa.int/web/guest/asset-database).

Koechlin et al. (2019) briefly described the CLIMSO instruments before focussing on image calibration. In this paper, we first give a thorough description of the instruments and image processing, we describe the database and the products one can find there; then we describe the current and future evolutions of space-weather applications; and at last, we introduce a new instrument under development.

\section{CLIMSO instrumentation and image processing}

To achieve the monitoring of the solar activity, CLIMSO observes the Sun through four instruments installed on the same equatorial mount: two solar refractors and two coronagraphs. (An overview of the instruments characteristics and products is given Table 1.) A fifth telescope, using a Fresnel diffractive array, is used for auto-guiding. This latter system is described by Koechlin et al. (2019).

\subsection{L1 and L2 solar refractors}

The two solar refractors L1 and L2 observe respectively the $\mathrm{H} \alpha$ and $\mathrm{CaII} \mathrm{K}$ line. We recall that the core of the $\mathrm{H} \alpha$ line images the middle chromosphere, while the upper photosphere (K1), the middle chromosphere (K2) and the upper chromosphere (K3) contribute to the CaII K line (Vernazza et al., 1981).

L1 (standing for "lunette 1", French for "refractor 1") is a $150 \mathrm{~mm}$ aperture refractor designed at Institut de Recherche en Astrophysique et Planétologie (IRAP). Its optical arrangement and mechanical configuration are shown in Figures 1 and 2, respectively. L1 comprises an iStar achromatic lens as objective (O1), and a Daystar $\mathrm{H} \alpha$ filter (F) centred on $656.28 \mathrm{~nm}$ with a bandwidth (Full Width at Half Maximum [FWHM] $)$ of $0.05 \mathrm{~nm}$. Then $\mathrm{O} 2$, which is a divergent-meniscus lens allows the beam to attack the filter $F$ at an $f / 30$ aperture ratio. This filter is based on a Fabry-Perot design. Its spectral transmission has wings that let some continuum from both sides of the solar $\mathrm{H} \alpha$ line to "leak" and somewhat reduce the contrast. A $0.04 \mathrm{~nm}$ wide $\mathrm{H} \alpha$ filter should replace it in the near future. 


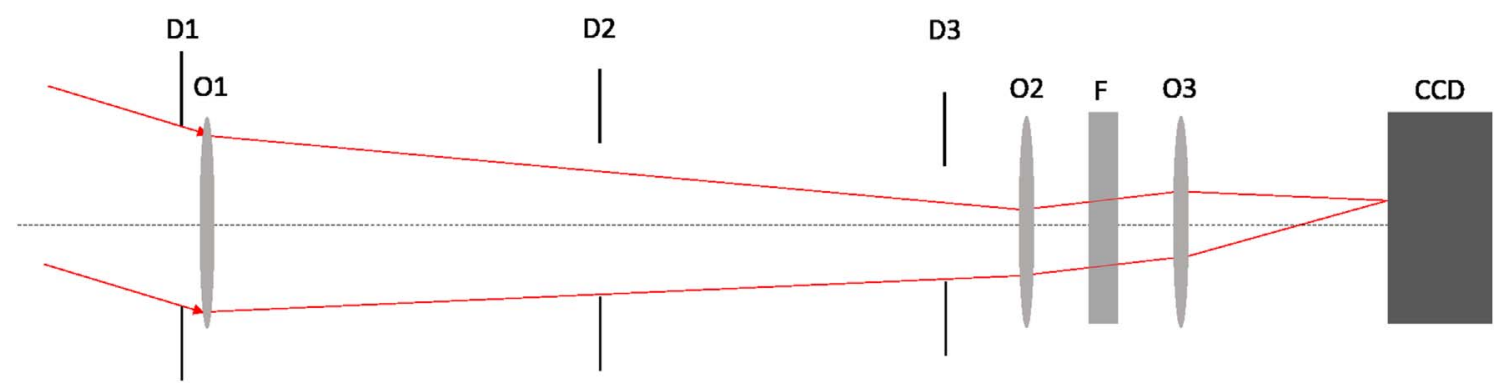

Fig. 1. Schematic of L1 refractor's optics.

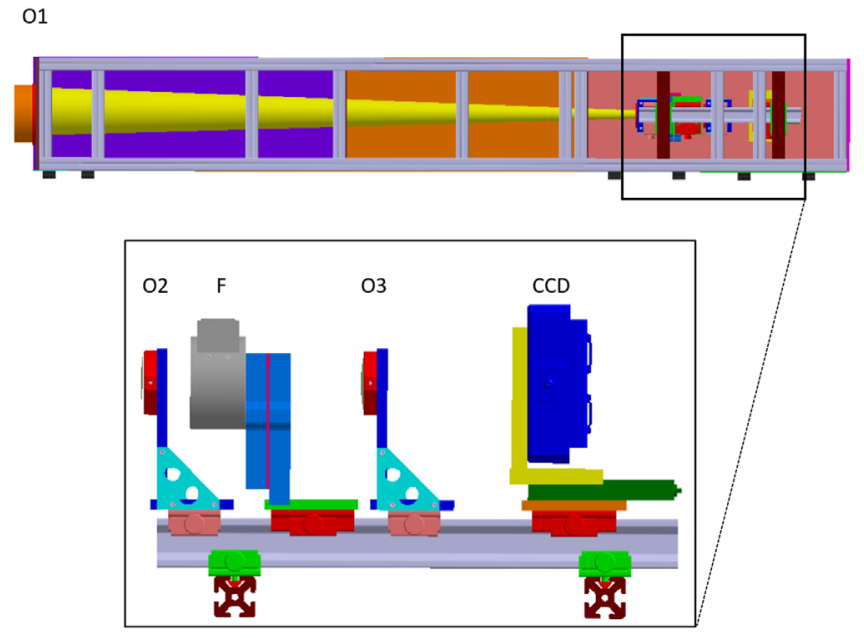

Fig. 2. Mechanical configuration of the $\mathrm{L} 1$ refractor with a zoom on its back end.

At last, $\mathrm{O} 3$ forms a image on the sensor. The lens set $(\mathrm{O} 2$ and O3) is optimised to reduce aberrations below the diffraction radius in most of the field. The lens combination adjusts the field allowed by the detector to 1.3 solar diameter. The $\mathrm{H} \alpha$ filter is mounted on a tilt plate. Although this feature has not been used yet, it is planned to allow for a fast scan of the solar $\mathrm{H} \alpha$ line. L1 is used to image the full disk of the sun (top left panel of Fig. 3) and essentially to capture and watch the evolution of filaments (Roudier et al., 2018). L1's resulting effective focal length is $1350 \mathrm{~mm}$. All lenses are made of BK7 glass. The details of the optical arrangement are given in Table 2. Let us emphasize that before 2014, L1 was a standard 90-mm Coronado telescope.

L2 is a $90 \mathrm{~mm}$ aperture refractor based on a Coronado standard model, the effective focal length of which was increased to $1250 \mathrm{~mm}$ (instead of $800 \mathrm{~mm}$ originally) with a Barlow lens. It is equipped with a CaII $\mathrm{K}$ filter, made by BARR, centred on $393.37 \mathrm{~nm}$ with a bandwidth (FWHM) of $0.25 \mathrm{~nm}$. This filter is therefore centred on the K3 line but with its wide bandwidth, the whole $\mathrm{K}$ domain contributes to the images. The $\mathrm{CaII} \mathrm{K}$ line is very sensitive to magnetic activity (top right panel in Fig. 3). In particular, brightening of the CaII K line in emerging flux region is a signature of arch filament foot points and, for extreme intensification, a signature of microflares (Pariat et al., 2007). Depending on the solar activity, the K1, $\mathrm{K} 2$ and K3 components contribute differently to the overall image (Oranje, 1983).

Both refractors are equipped with a CCD camera (APOGEE Alta U4000). The sensor comprises $2048 \times 2048$ pixels, each of which is $7.4 \mu \mathrm{m}$ in size. Their absolute quantum efficiencies at $656.28 \mathrm{~nm}(\mathrm{H} \alpha)$ and $393.37 \mathrm{~nm}(\mathrm{CaII} \mathrm{K})$ are about $30 \%$ and $40 \%$, respectively. These cameras are being replaced by ANDOR Zylas (see next section). Angular resolutions of the order of 1 arcsec is frequently achieved before noon at Pic du midi, when the sky is clear.

\section{$2.2 \mathrm{C} 1$ and $\mathrm{C} 2$ solar coronagraphs}

Coronagraphs $\mathrm{C} 1$ and $\mathrm{C} 2$ allow the imaging of solar prominences on the limbs through $\mathrm{H} \alpha(656.28 \mathrm{~nm})$ and neutral helium HeI $(1083.0 \mathrm{~nm})$ respectively. Both filters have a bandwidth (FWHM) of $0.25 \mathrm{~nm}$. All the optics found themselves in two aluminium structures of octogonal cross-section. Each of them comprises two lenses and two groups of lenses, an occulting disk (Lyot mask), several diaphragms including the Lyot stop, and a band-pass filter that selects a given wavelength. The optical principle, shown in Figure 4, is the following.

The objective (plano-convex) lens O1, stopped down by diaphragm D1, produces a real image of the Sun in its focal plane. In this focal plane, an occulting disk (Lyot mask, LM) is placed to block the light coming from the solar disk. The shape of the occulting disk was designed to be the continuation on a cone-shaped mount (Fig. 5) that contribute to reject the light and heat outward. Then, the lens $\mathrm{O} 2$ plays two roles: it forms a real image of D1 in a plane where the Lyot stop (LS) is placed. To remove light undergoing two reflexions inside $\mathrm{O} 1$ and forming a parasitic image of the solar disk some millimetres behind the centre of LS, a tiny mask is added there. Next, the doublet O3 creates an image of the occulted Sun at infinity so that all rays of light from a given source point on the corona go through the filter $(\mathrm{H} \alpha$ or $\mathrm{HeI})$ with the same incident angle. At last, the filtered light goes through the doublet O4 that forms a real image of the occulted Sun in its focal plane where the camera is placed (Fig. 6). The characteristics of each elements are given in Table 3 . In addition to this optical arrangement, baffles are placed to block as much as possible all sources of stray light. More details on the optics and in particular how to 


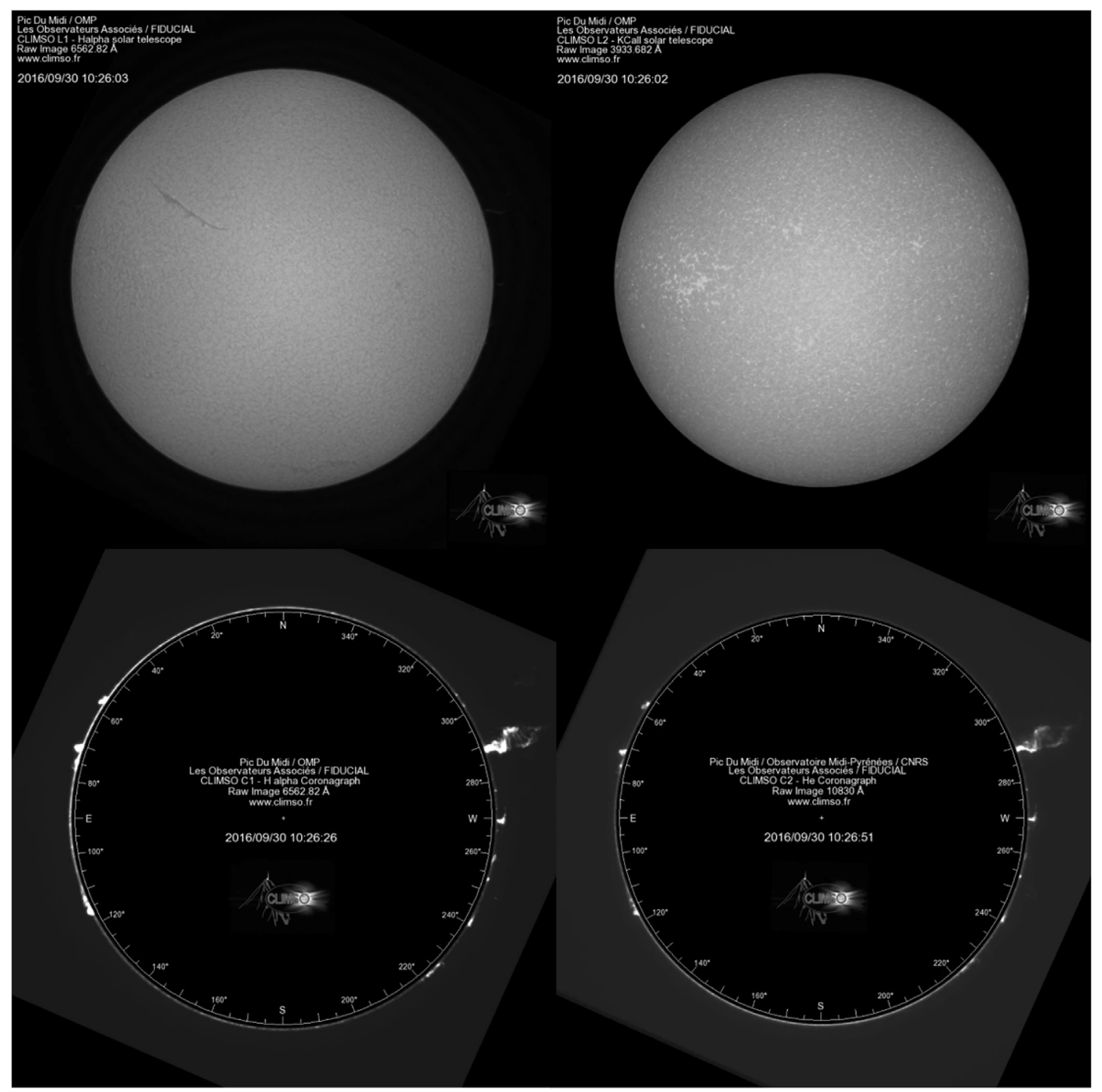

Fig. 3. Images from the four instruments taken on 30 September 2016 around 10:26 UT. The L1 H $\alpha$ refractor is on the top left panel; L2 CaII K refractor on top right; $\mathrm{C} 1$ coronagraph in $\mathrm{H} \alpha$ at the bottom left; and $\mathrm{C} 2$ coronagraph in $\mathrm{HeI}$ at the bottom right.

get rid of ghost images and how to deal with aberrations can be found in Dettwiller \& Noëns (2008).

For both $\mathrm{C} 1$ and $\mathrm{C} 2$, the focal length of $\mathrm{O} 1$ is of the order of $2500 \mathrm{~mm}$ (Table 3). The Lyot masks are changed about every ten days to adapt their sizes to the changing apparent diameter of our Sun. For C1 (C2), the diameter of LM ranges from 22.60 (23.05) mm early July, when the Sun-Earth distance is at its maximum, up to 23.40 (23.80) $\mathrm{mm}$ in early January when the Sun is at its closest.

Both $\mathrm{C} 1$ and $\mathrm{C} 2$ are equipped with an ANDOR Zyla 4.2 camera with a CMOS sensor. Its resolution is $2048 \times 2048$ and its pixel size is $6.5 \mu \mathrm{m}$. Quantum efficiency in visible at $656.28 \mathrm{~nm}(\mathrm{H \alpha})$ is slightly below $80 \%$, while at $1083.0 \mathrm{~nm}$ (HeI) it is lower than $1 \%$. (Obviously, this camera was not designed for capturing near infrared photons.) Instances of pictures taken by $\mathrm{C} 1$ and $\mathrm{C} 2$ are shown in Figure 3 (bottom left and right panels respectively).
Table 2. Optical specifications of L1.

\begin{tabular}{lc}
\hline & L1 (mm) \\
\hline Aperture of O1 & 150 \\
Focal length of O1 & 2250 \\
Distance O1 - O2 & 1850 \\
Focal length of O2 & -745 \\
Distance O2 - F & 103 \\
Distance F - O3 & 103 \\
Focal length of O3 & 252 \\
Distance O3 - camera & 174 \\
Focal length of the whole instrument & 1350 \\
\hline
\end{tabular}

In addition to the snapshots shown in Figure 3, we have added a movie as a supplementary material. It is made out of the images of L1 and C1 taken on 14 December 2010 and shows the ejection of a filament. 


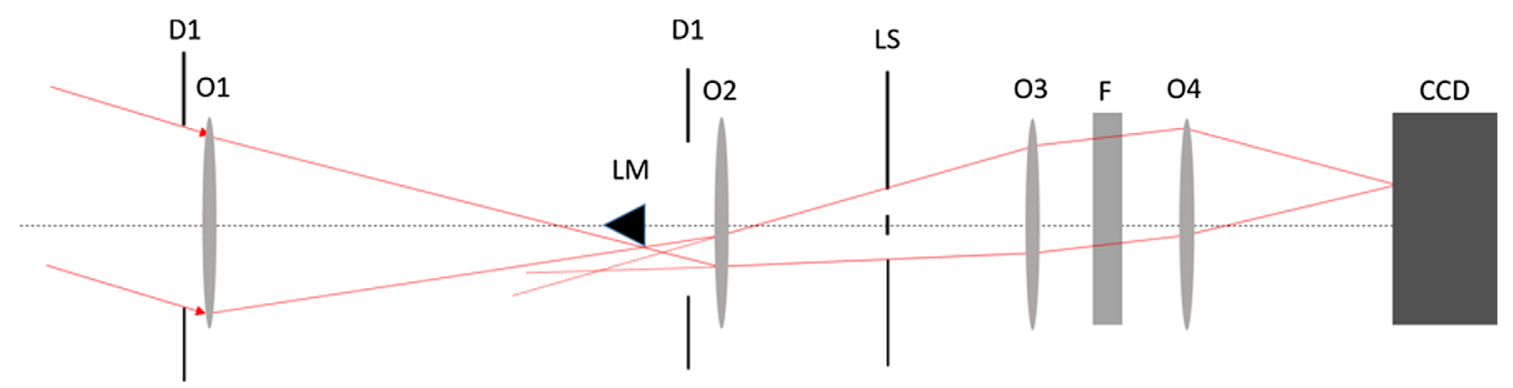

Fig. 4. Schematic of the coronagraphs' optics with the paths of two rays of sunlight. (Adapted from Dettwiller \& Noëns, 2008).

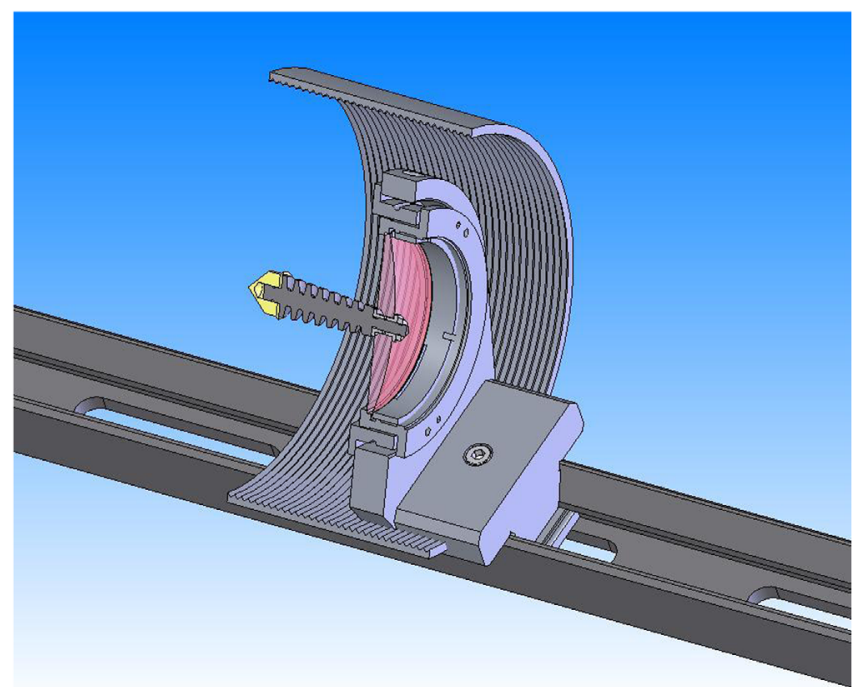

Fig. 5. O2 lens (in pink) carrying the mount of the occulting disk and cone (in yellow).

\subsection{Processing and photometric calibration}

To process the images, flat fields, dark and bias frames are taken once a day. CLIMSO is equipped with a $1350 \mathrm{~mm}$-wide diaphragm wheel in front of the instruments (Fig. 7). The holes in the wheel have been placed so that each of the four instruments can be aligned with its dedicated slots where holes were pierced or filter were placed (see details in Fig. 8).

First, in the early morning, the "Point" position is used for pointing the Sun and initiate the autoguiding system. The dedicated slots are pierced with three 10 -mm wide holes that are placed $120^{\circ}$ from one another on a $150-\mathrm{mm}$ wide circle. If the systems are in-focus, the three holes form a single image of the Sun (L1 and L2) or of the corona (C1 and C2).

Then, for observations, the full-aperture "Obs" diaphragms are placed in front of the instruments.

The "Flat" full aperture diaphragms are filled with ground glass and are used for the flat fields.

Also, once a day, two consecutive images are made at 1-min interval through the "Cal" holes $(10 \mathrm{~mm}$ across) while the equatorial drive is stopped, letting the Earth rotation displace the solar disk image. Correlating these two images yields the vector. The orientation files give the $\mathrm{E}-\mathrm{W}$ axis: they go by pairs, one pair each day for each instrument. This procedure, combined with the Carrington ephemeris giving the apparent

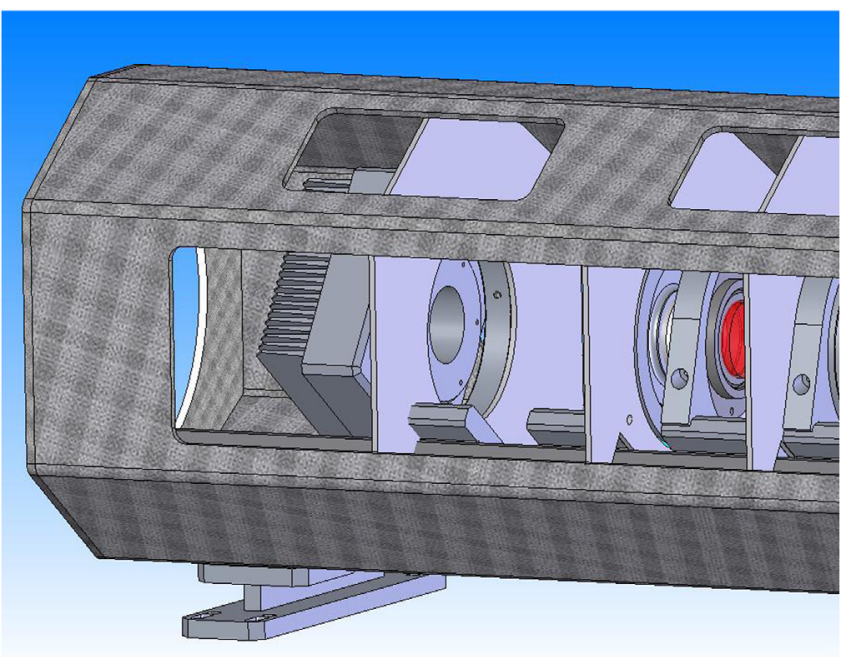

Fig. 6. Detailed view of the $\mathrm{C} 1$ back end in its hexagonal structure. From right to left one can see the Lyon stop hold by the red panel, $\mathrm{O} 3$, the $\mathrm{H} \alpha$ filter, $\mathrm{O} 4$, and at the far left the $\mathrm{CCD}$ camera.

Table 3. Optical specifications of $\mathrm{C} 1$ and $\mathrm{C} 2$.

\begin{tabular}{lcc}
\hline & C1 $(\mathrm{mm})$ & C2 $(\mathrm{mm})$ \\
\hline Aperture of O1 & 200 & 200 \\
Focal length of O1 & 2456 & 2488 \\
Distance O1 - LM & 2456 & 2488 \\
Distance LM - O2 & 56.5 & 56.5 \\
Focal length of O2 & 300 & 300 \\
Distance O2 - LS & 341 & 340 \\
Aperture of LS & 22 & 20 \\
Distance LS - O3 & 68 & 68 \\
Focal length of O3 & 486 & 500 \\
Distance O3 - F & 60 & 40 \\
Distance F - O4 & 60 & 40 \\
Focal length of O4 & 200 & 200 \\
Distance O4 - camera & 200 & 195 \\
Focal length of the whole instrument & 1244 & 1133 \\
\hline
\end{tabular}

inclination of the Sun rotation axis, allows us to orient the images of the Sun with its North pole on top.

During the night, dark and bias frames are automatically done. Let us note that dark files and bias files are quasi equivalent, as the exposure times are very short (see the fits headers of these files). 


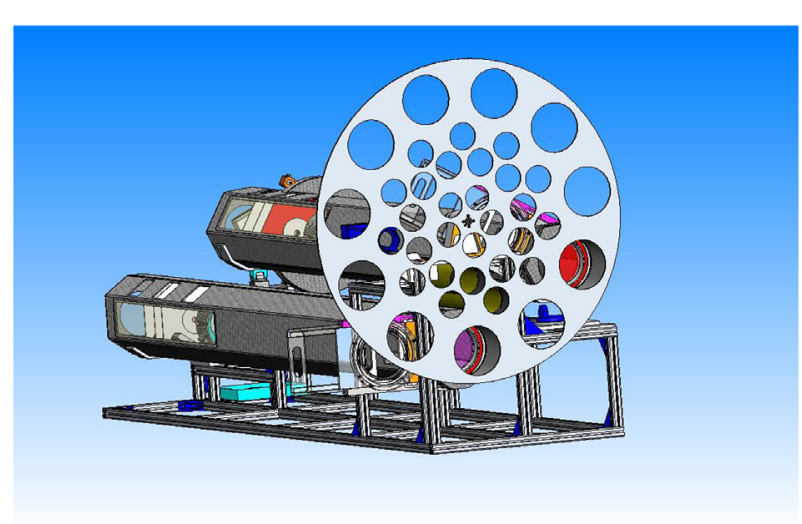

Fig. 7. $\mathrm{C} 1$ and $\mathrm{C} 2$ coronagraphs and their calibration wheel.

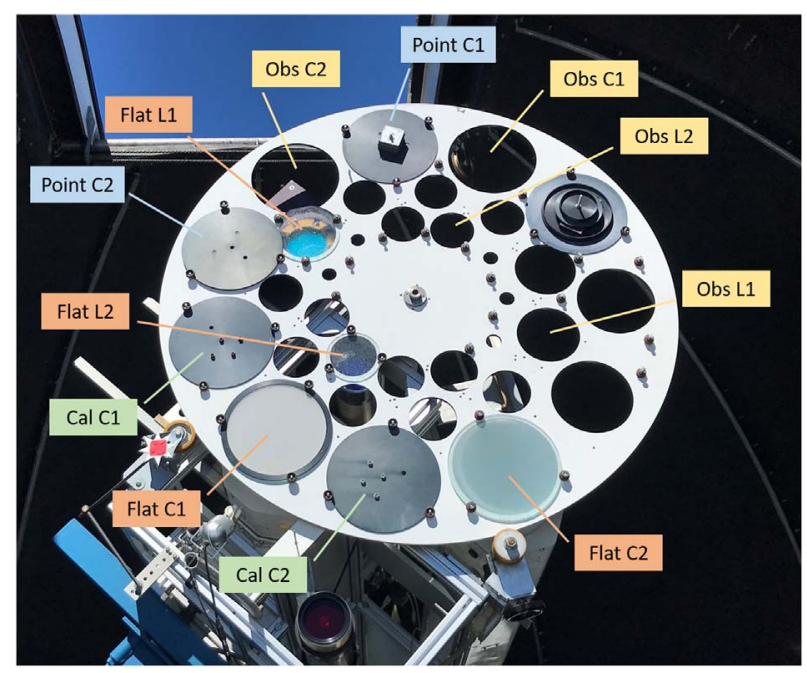

Fig. 8. CLIMSO's front filter wheel with the functions of the used slots for pointing the Sun ("Point" blue labels), observations ("Obs" yellow labels), calibration ("Calib" green labels) and flat fields ("Flat" orange labels).

At this stage, all $\mathrm{C} 1$ and $\mathrm{C} 2$ processed images are called "calibrated": there are corrected from the flat field, from the dark and bias frames, and there are oriented.

For L1 and L2, the calibration process goes further as we perform a photometric calibration: we offer images in physical units. Each pixel of the image corresponds to spectral radiance integrated over the spectral profile of the filters of L1 and L2, in $\mathrm{W} \mathrm{m} \mathrm{m}^{-2} \mathrm{sr}^{-1}$. The reader may find all the details of the calibration procedure in Koechlin et al. (2019).

\subsection{CLIMSO as a test bench: imaging the FeXIII line with coronagraph $\mathrm{C2}$}

To image the hot corona, some tests have been performed on C2 with a FeXIII filter (1074.7 nm, $0.25 \mathrm{~nm}$ FWHM) and a tip-tilt method to remove the blinding background. Practically, the FeXIII filter and the original HeI filter of $\mathrm{C} 2$ have been mounted on a motorised sliding rack, which allows switching from one filter to the other in a few seconds. On some images and after thorough processing, some magnetic loops of the hot low corona appear (Fig. 9).

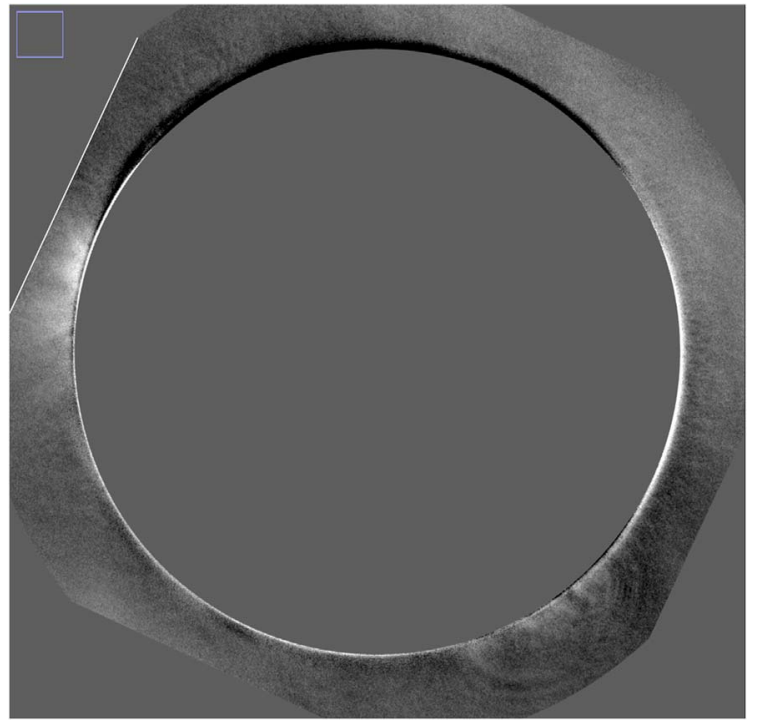

Fig. 9. Image from C2 with FeXIII filter on 17 September 2017 at 10:57 UTC. Magnetic field lines are visible almost all around the solar disk (magnetic loops on the top left and bottom right for instance).

These tests were only partly conclusive for two main reasons. First, our camera is not designed to capture near-infrared photons (the quantum efficiency at $1074.7 \mathrm{~nm}$ is below 1\%). This very low quantum efficiency requires long exposure times (typically 10 times $0.6 \mathrm{~s}$ : $0.3 \mathrm{~s}$ centred on the line followed by $0.3 \mathrm{~s}$ away from it for sky background subtraction), which is far too long. The second reason is that the $1074.7 \mathrm{~nm}$ filter alone is not able to achieve the high dynamic range needed to image the corona: it had leaks in the visible. In 2016, we completed the original design with an infrared filter that blocks the wavelengths shorter the $850 \mathrm{~nm}$ and a thorough baffling. A post processing based on statistics over 10 exposures of $300 \mathrm{~ms}$ each completed the setup. The post-processing algorithms developed in 2015 and 2016 are coded in Python and $\mathrm{C}++$, they are publicly available. The FeXIII line we detect is ten to hundred times weaker than $\mathrm{H} \alpha$ in prominences and barely brighter than the sky background: typical values we obtain are about 2200 ADU in the prominences and 1500 ADU in the sky background. This optical setup and the software allowed us to carry a survey of the Fe XIII corona over a couple of years.

To the authors's knowledge, the imaging of the FeXIII line from the ground is only performed on a regular basis at the Mauna Loa Solar Observatory (MSLO) on Hawaii and at CLIMSO. The reader should note that the two observatories are located $156^{\circ}$ apart in longitude, which maximizes the temporal coverage of solar observations. In other words, the two sites and their instruments complement each other very nicely.

\section{Operations and data archiving}

\subsection{Operations}

The Observateurs associés run all the observations. They go up to the observatory by pairs for a week, from a Monday to the next. In principle, 50 full weeks (i.e., 350 days) a year are covered. In practice, the statistics of actual observation are lower: 


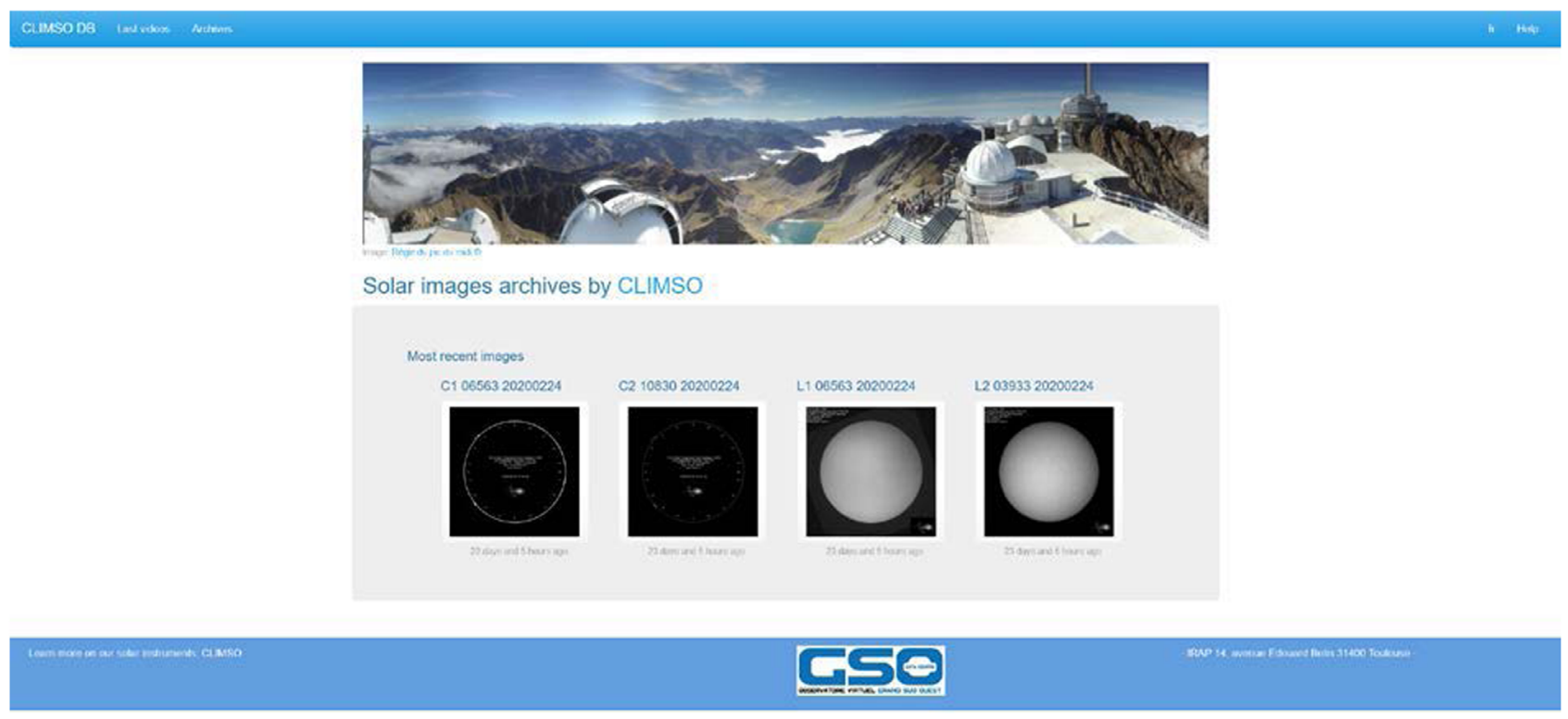

Fig. 10. Portal of the Climso-DataBase web site (http://climso.irap.omp.eu).

over the years 2009-2019, observations were made 153 days per year on average (min. 123; max. 186). The main reason for this relatively low number is the weather conditions of course; maintenances may also occur.

In routine mode, the four instruments take one image every minute. When operational during its test phase, images of $\mathrm{C} 2$ in FeXIII mode were taken every hour. But lately, we have been observing the Sun in coordination with Parker Solar Probe (PSP) around its perihelia, for which higher cadence have been required. (Likewise, we plan some joint observations with Solar Orbiter.) We thus envision increasing the acquisition cadence to approximately one image every $10 \mathrm{~s}$ or $15 \mathrm{~s}$ for limited timeperiods, on demand or on alert. These higher cadences are feasible but require higher memory capacity and a better synchronisation between all instruments. In fact, the camera of each instrument is controlled by its own computer whose reaction time in driving the camera may differ from the others. As a result, although the command that triggers the captures is sent simultaneously every $60 \mathrm{~s}$ to the four computers, the actual image time tags may be off by several seconds. This is assumably a minor problem for operational space weather applications but it is a problem for solar physics, especially when it comes to accurately time tagging transient phenomena and comparing images from our four instruments. These two points (higher memory capacity and better synchronisation) will be taken care of in the course of 2020 or beginning of 2021.

\subsection{CLIMSO database}

All individual images and movies are sent every night to the CLIMSO database (called CLIMSO-DB) at IRAP, Toulouse (https://climso.irap.omp.eu). CLIMSO-DB is part of the Grand Sud-Ouest Virtual Observatory (OV-GSO; Sanguillon et al. 2019)

Practically, there are several options from the portal (Fig. 10). The user may visualise the latest images displayed on the page and possibly select them for larger version of them. One may click the top left link "Latest videos" and access to the latest videos from the four instruments). There, at the bottom of the page, so-called combined videos, that are superimposed movies from $\mathrm{L} 1$ and $\mathrm{C} 1$ (full disk and prominences in $\mathrm{H} \alpha$ ), are available.

Still from the portal, the user may also access the full archive from the top left link "Full archive" in the blue top banner. The user then ends up on the page shown in Figure 11 (http://climso.irap.omp.eu/data/archives.html). There, all pictures and movies are stored. They may be visualised or downloaded as JPG pictures, MP4 movies or FITS files. On the left part, one may select the instruments, the level of analysis (raw or calibrated images) or the line. On the central frame, dates are sorted by month ("month" tab) or all dates are shown year after year, month after month ("all dates" tab).

The syntax used for files names is the following: imoa_<wavelength $\rangle_{-}<$instrument name $\rangle_{-}<$date $\rangle_{-}<$time $\rangle_{-}$ $<$ file type $>$.fts

For each date, calibrated images are available and one should note that this has a different meaning depending on the instruments. For L1 and L2, calibrated images are photometrically calibrated as explained by Koechlin et al. (2019) and the corresponding files are named *_emil.fts. Regarding $\mathrm{C} 1$ and $\mathrm{C} 2$, this photometric calibration is not done yet; the so-called calibrated images are just oriented and corrected for the dark, flat, and bias frames. They are named * bril.fts.

For those who would like to do the calibration themselves, the raw images and the calibration files are also at disposal with the following naming (file type):

- Raw images: *_bl.fts

- Dark files: *_da.fts

- Flat files: * pl.fts

- Bias files: * bi.fts

- Orientation files : *_ca.fts.

Apart from its dedicated database and website, CLIMSO data are reachable through web services. 


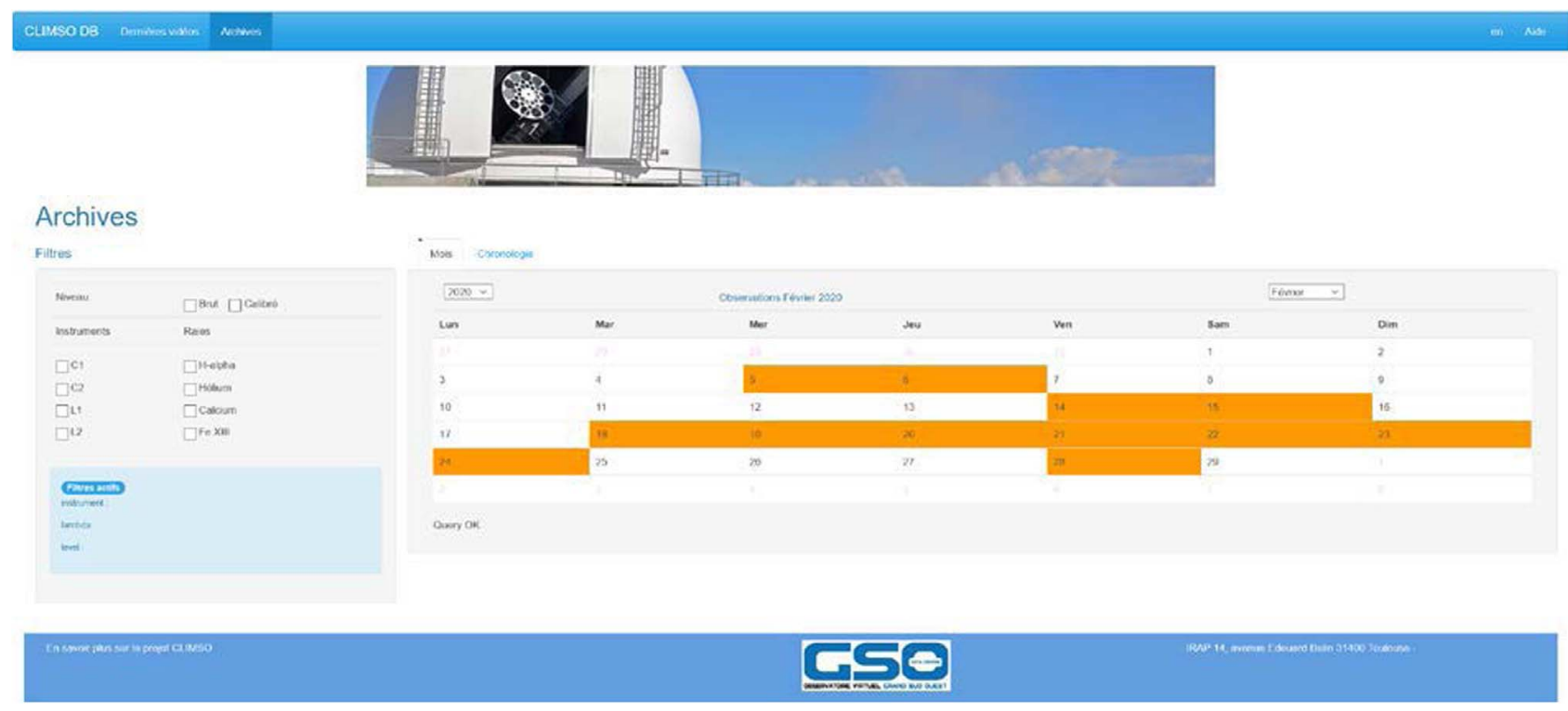

Fig. 11. Page of the full CLIMSO archive (http://climso.irap.omp.eu/data/archives.html).

CLIMSO data are accessible from the Virtual Solar Observatory (Hill et al., 2009): https://sdac.virtualsolar.org/cgi/search.

The CLIMSO web service is also registered on the International Virtual Observatory Action (IVOA) registry. The database is thus accessible to any client able to query the IVOA registry through the VO-compatible EPN-TAP protocol. The protocol and the underlying infrastructure are detailed by Erard et al. (2014). To this end, the CLIMSO DB's endpoint is: http://climso.irap.omp.eu/_system_/tap/run/tap/sync where a request can be placed. For instance, if one wishes to retrieve the 10 first data the CLIMSO's EPN-TAP service finds: http://climso.irap.omp.eu/_system_/tap/run/tap/sync?LANG= ADQL\&QUERY=SELECT +*FROM+climso.epn_core+\& REQUEST $=$ doQuery \&MAXREC $=10$. This command line returns a VO-Table with the requested information. (VO-Tables can be read with a tool such as the Tool for Operations on Catalogues And Tables (TOPCAT; Taylor, 2005) for instance.

CLIMSO parameters are named climso.epn.core.*, the star (*) being any of the available EPN-TAP parameters (https://voparis-wiki.obspm.fr/display/VES/EPN-TAP+V2.0 +parameters).

Any database or service making use of the EPN-TAP protocol, like the Automated Multi Dataset Analysis (AMDA; Jacquey et al., 2010; Génot et al., 2020) or the Virtual European Solar and Planetary Access (VESPA; Erard et al. 2018) for instance can thus access the CLIMSO database. From AMDA, one should use the link "Interoperability" on the bottom right of the work space (Fig. 12) while from VESPA, CLIMSO is suggested in the main page as a possible "EPN resource".

All VO applications can be found on the IVOA web page: http://www.ivoa.net/astronomers/applications.html. The reader also should note that CLIMSO-DB can be accessed directly through, and the VO-tables handled with, commands written in Astronomical Data Query Language (ADQL; http://www. ivoa.net/documents/ADQL/).

At last, CLIMSO data are open; their use is ruled by the creative commons licence CC-BY-NC-SA 4.0, unless further notice.

\section{On-going developments for space weather applications}

Software developments are underway to better meet the needs of the solar and space weather communities in term of swift data access and to improve the visibility and valorisation of CLIMSO images. In addition, on the instrumentation side, a new coronagraph is under development.

\subsection{Real time access}

Real-time images of the full disk in $\mathrm{H} \alpha$ and CaII $\mathrm{K}$, as those provided by CLIMSO's L1 and L2, are of first importance as they allow watching after the solar active regions. The brightening of those regions may be indicative of an on-going solar flare or a flare to come. Filaments are also features to be looked after as they can take off anytime and be the precursor of a CME. In fact, it was shown by Yurchyshyn et al. (2003) that the most geoeffective CMEs originate within $0.6 R_{\mathrm{S}}$ of the centre of the solar disk. Another interesting feature to observe for space weather are chromospheric Moreton waves (Moreton, 1960). They are seen in $\mathrm{H} \alpha$ and, while there have been claims that they could be a precursor for CMEs, it is well reported that they are associated with type-2 radio bursts (Cliver et al., 1999) and may be a signature of on-going flare and CME (Chen et al., 2005; Chen, 2011).

As far the coronagraphs are concerned, they provide images that are used to watch the prominences and magnetic loops. When those are ejected, it may be the sign of a larger type of event and their speed gives an idea of a CME velocity in its early stage. (The initial speed of a CME, compared to its speed measured further away from the Sun, enables the calculation of the acceleration or deceleration of the structure, and therefore to have a more accurate estimate of the arrival time at 1 AU.) Space-borne coronagraphs, since they are constantly moving, give with difficulty access to the very low chromosphere and corona and thus to this early stage velocity. Due to this, with their field starting very close to the solar disk (at 1.001 solar 


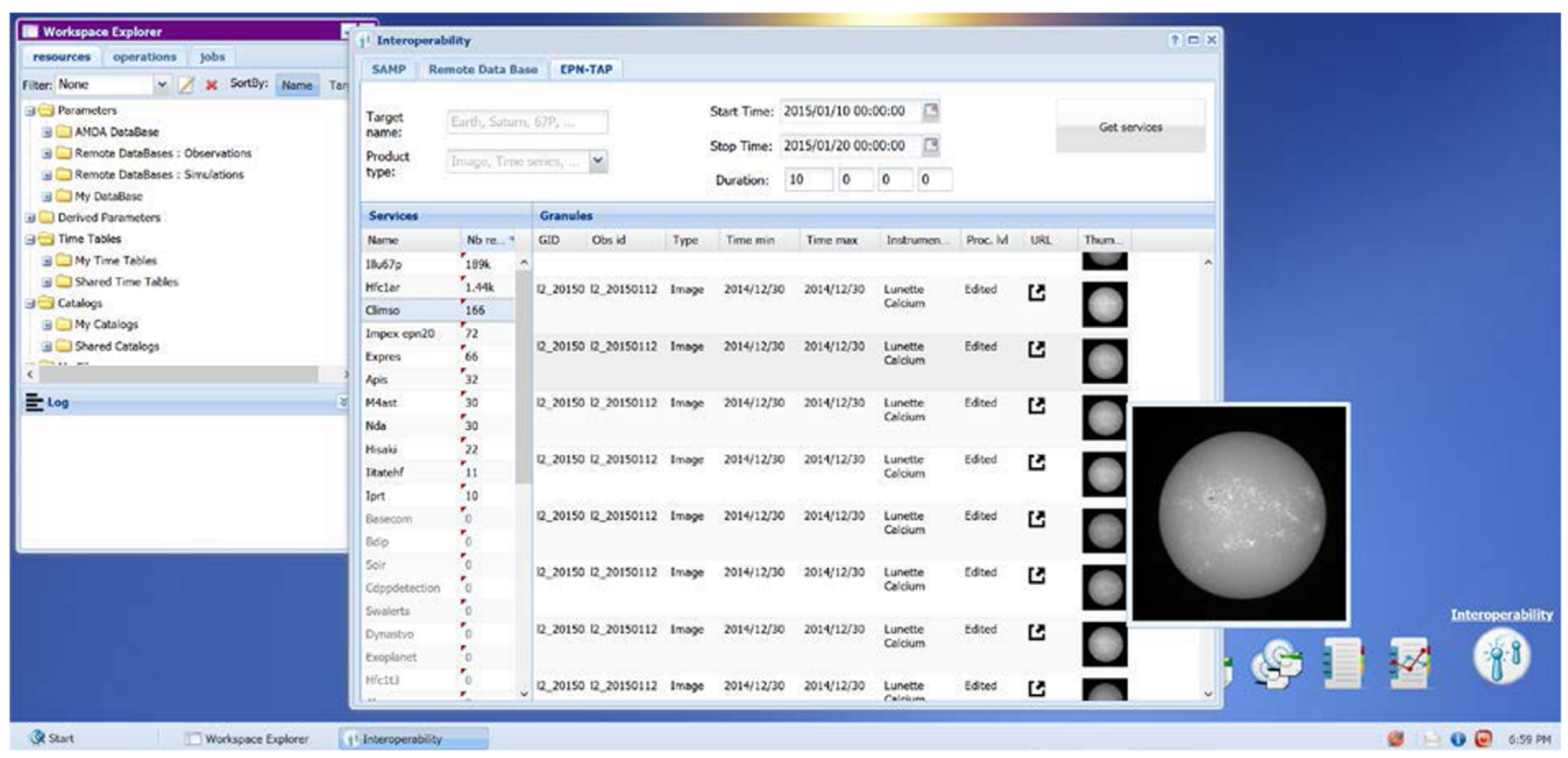

Fig. 12. CLIMSO archive visible and accessible from AMDA through EPN-TAP web service.

radius), ground based coronagraphs complement very well space solar telescopes. Besides, as the weather is not always ideal (an average of 153 days of observations per year at Pic $\mathrm{du}$ Midi), ground-based solar telescopes and coronagraphs also complement one another.

Now, the acquisition procedures and the back end of the CLIMSO system were not initially designed to make the images available to all users in real time. For the moment, apart from the CLIMSO operators that have a real-time access to the images, the latest CLIMSO images are only accessible a few hours after sunset, i.e., about 22 UTC depending on the season. The only way of obtaining real-time images is to be granted a special and direct access to the computer that collects the images. To date, this special access was given only once (to the space weather service of the French Air Force). We have had requests and suggestions for a more rapid access to the images, even not calibrated, for real-time solar surveillance. We are thus in the process of sending the latest raw (uncalibrated) images to CLIMSO-DB and of making them available (almost) as soon as they are taken. Those raw images are good enough to spot the brightening of active regions, the take-off of some filament or prominence. This should be done by the end of 2020 .

\subsection{Data input for and from the STORMS national service}

STORMS is the Solar Terretrial ObseRvation and Modelling Service developed by and hosted at IRAP (http://stormsservice.irap.omp.eu/). The goal of the service is, among other things, to study the propagation of solar properties with the solar wind through the heliosphere. In particular, the STORMS service will ultimately predict the IMF and solar wind properties at $1 \mathrm{AU}$ and also predict geomagnetic indices. To achieve this, the service needs its propagation models to be fed with solar inputs, such as Carrington maps of the solar disk in $\mathrm{H} \alpha$ or
Carrington maps of the limb. CLIMSO is able to provide these inputs and we are working at it.

Since 2020, CLIMSO has joined the group of groundbased instruments that make complementary observations to cover the PSP perihelia passages (https://whpi.hao.ucar.edu). On this occasion and at the Whole Heliosphere and Planetary Interactions (WHPI) community's request, we have restarted measurements with the FeXIII filter of C2. It is partly this community that suggested that we should increase our acquisition cadence. We had in mind to increase the cadence during the fifth encounter early June 2020 but the Covid-19 pandemic delayed our plans.

As part of the STORMS national service, the magnetic connectivity tool (http://connect-tool.irap.omp.eu/) determines PSP's, Solar Orbiter's and Stereo A's magnetic and radial footprints on the solar surface (Fig. 13). The aim is evidently to link their measurements to solar features on the photosphere and chromosphere. We plan to include these footprints on CLIMSO images. Still in link with PSP encounters and Solar Orbiter observations, but outside the scope of the present paper, the Pic du Midi observatory has also a 500-mm Jean Rösch refractor equipped with a wide-field camera (about a third of the solar surface covered). Once the PSP and Solar Orbiter footpoints on the solar disk are predicted, we can point this instrument to the region of interest. The goal is to observe the activity on the solar photosphere such as sunspot changes, which are indicative of modifications in magnetic topology (Wang et al., 2013) that could occur during, and in link with, in situ observations by the solar probes.

\section{A third coronagraph under study}

In Section 2.4, we mentioned the tests we performed to image the magnetic loops within the corona. For the reasons put forward, those tests were only partly satisfactory. To remedy 


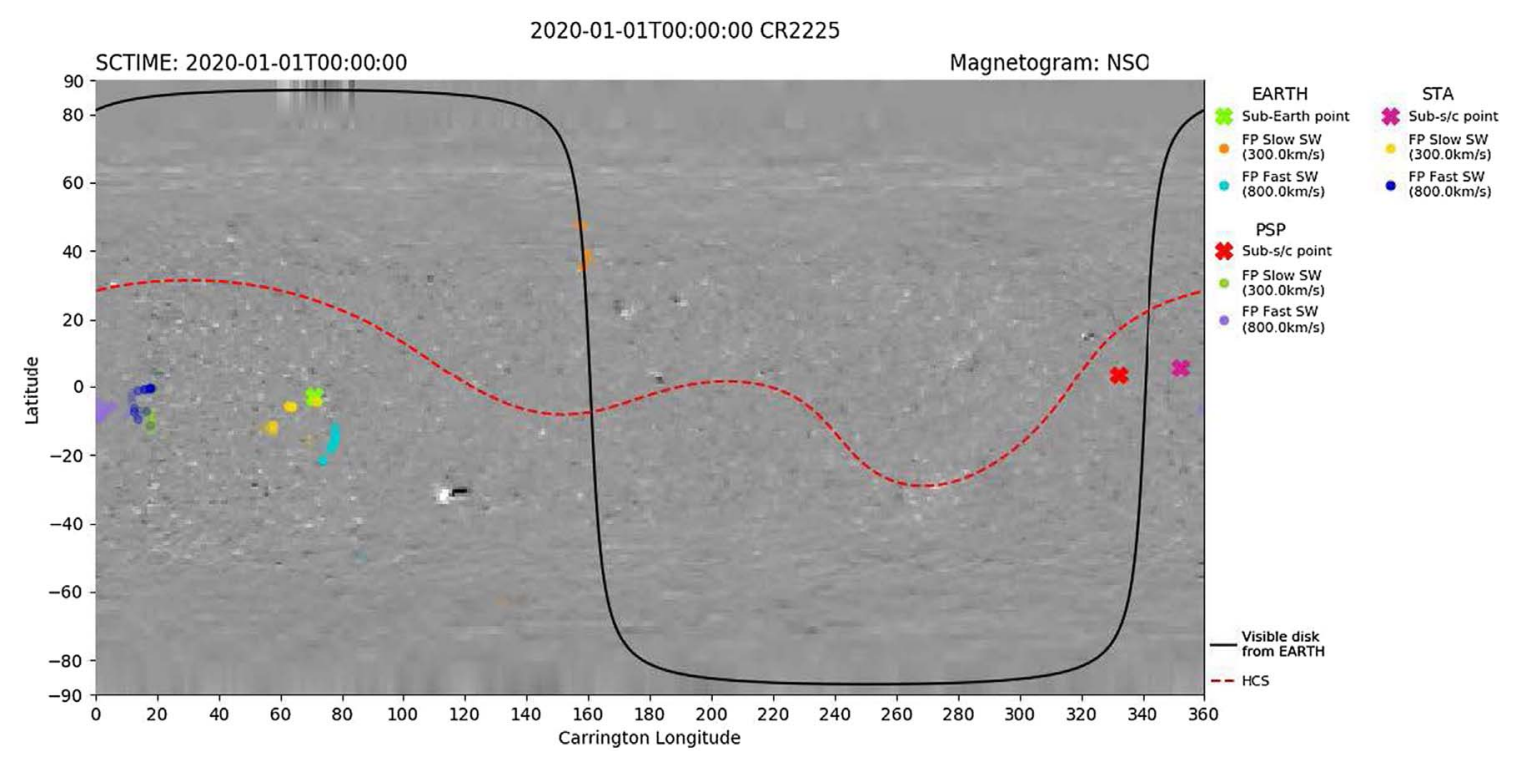

Fig. 13. Output from the Magnetic connectivity tool showing, superimposed on some Carrington map, the expected locations of the Earth, Parker Solar Probe (PSP), and Stereo A (STA), for slow and fast solar wind.

the problems raised, we are in the process of designing a third coronagraph - called $\mathrm{C} 3$ - entirely devoted to the observation of the hot and low corona. $\mathrm{C} 3$ will have polarimetric capabilities: a modified Lyot or an apodized Šolc filter will enable the measurement of the $Q$ and $U$ Stokes parameters.

It will observe the FeXIV green line at $530.3 \mathrm{~nm}$. This choice is motivated mainly by a cost/performance ratio: an infrared camera to observe the FeXIII line in good conditions is outside our budget. Besides, the FeXIV line shows rates of polarisation of $1-3 \%$, with peaks up to $10 \%$, which are measurable. At last, observing a different line than at MSLO (FeXIII) could be advantageously complementary.

With this instrument, we shall thus be able to measure the velocity and magnetic field inside magnetic loops and contribute to unveiling heating processes such as superoscillations (López Ariste \& Facchin, 2018). Such filters are not very luminous and as we aim at observing a line whose radiance is $10^{-5} \times \mathrm{S}_{530.3}$ $\left(\mathrm{S}_{530.3}\right.$ being the radiance of the solar disk at $\left.530.3 \mathrm{~nm}\right)$ with an expected sensibility of $10^{-6} \times \mathrm{S}_{530.3}$, we need a larger aperture than $\mathrm{C} 1$ and $\mathrm{C} 2$. Given all those needs, the foreseen specifications of the instruments are the following:

- Aperture: $400 \mathrm{~mm}$ diameter

- Focal length of the aperture lens: $3200 \mathrm{~mm}$ (F/8)

- Field of view: 2.6 solar radii

- Resolution: 2.4 arcsec/pixel with $2048 \times 2048$ camera; 1.2 arcsec/pixel with a $4 \mathrm{~K}$-camera

- FeXIV filter: $530.3 \mathrm{~nm}$ (with spectral resolution better than $0.1 \mathrm{~nm}$ on a $\pm 30 \mathrm{~km} / \mathrm{s}$ range).

The filter is still under investigation. It will have (i) to select the wavelength of $530.3 \mathrm{~nm}$ with a good spectral resolution, (ii) to allow us to tune the selected wavelength, and (iii) to be compatible with the measurement of the polarisation. Having these in mind, the filter will presumably have the following properties:

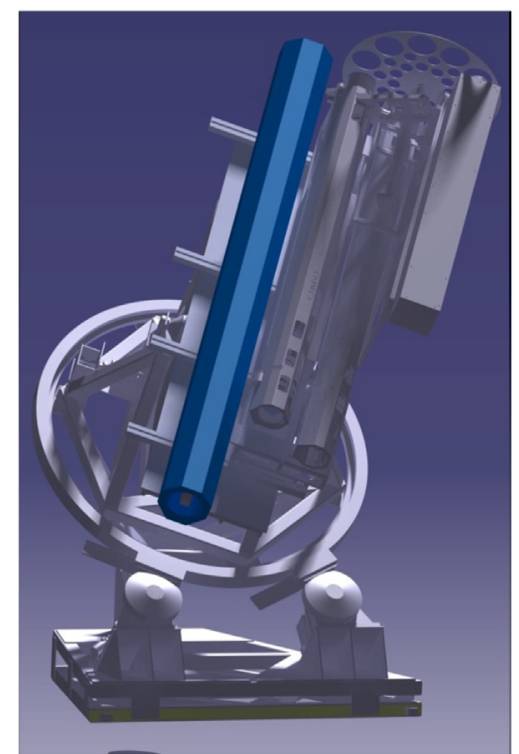

Fig. 14. 3D-model of the $\mathrm{C} 3$ coronagraph (in blue) by the other CLIMSO instruments' side.

- Type: modified Lyot or Šolc

- Material: $\mathrm{LiNbO3}$ and $\mathrm{TeO}_{2}$ are considered

- Aperture: $50 \mathrm{~mm}$

- Angle of incidence: $<3^{\circ}$.

The polarisation measurements will be performed thanks to the rotation of a linear polariser placed in front of the filter or to the rotation of the whole filter itself.

The $\mathrm{C} 3$ coronagraph will be installed on the same equatorial mount as the four other CLIMSO instruments (Fig. 14). Optical and mechanical design studies are underway. The main 
problems faced are the design of the filter and the birefringence, under mechanical stress, of the glass used for the lenses. (A lens becoming birefringent would alter the polarimetric measurements.)

We hope to have C3's first light in the course of 2021 or 2022 at latest. After a commissioning phase of a couple of months typically, it will be operational for fundamental solar physics research. Although no synoptic observations are planned, at first at least, measurements by $\mathrm{C} 3$ will allow us to have a precise idea of the magnetic field inside prominences before their possible take-off. This will be very useful to calculate the magnetic helicity and possibly, to predict the orientation of the interplanetary magnetic field inside magnetic clouds associated to coronal mass ejections. This orientation is one of the key parameters that control the coupling between the solar wind/IMF and the magnetosphere (Yurchyshyn et al., 2003).

\section{Concluding remarks}

Many ground-based solar coronagraphs have closed down in recent years. Yet, they are very valuable as they can capture the very low solar corona; they are much cheaper to build and maintain than space telescopes; and they can be used easily to test new techniques. CLIMSO offers a set of instruments that prove suitable for solar physics studies and solar surveillance. The four telescopes (two refractors and two coronagraphs) image respectively the full disk of our star and its near environment. The images and films are accessible through the CLIMSO-DB database and EPN-TAP compatible web services. On-going developments tend to turn CLIMSO into a real time solar monitor for space weather applications. In parallel, we are developing a third coronagraph that, on top of becoming one of the largest in the world, will complement our solar observations with more detailed imaging of the hot corona and polarimetric capabilities to infer the velocity and magnetic field inside magnetic loops. Besides, CLIMSO is a very good example of fruitful and effective pro-am collaboration. We do hope that the space weather community will find the CLIMSO system useful and we are open to any suggestion that would better meet their needs.

Acknowledgements. The Observateurs associés are funded by Fiducial. The CLIMSO national observation service is supported by CNRS/INSU, Observatoire Midi-Pyrénées, and Université Toulouse 3 - Paul Sabatier. The authors would like thank Jacques-Clair Noëns, the father of this project, and all the Observateurs associés for their devotion and seriousness in operating CLIMSO. Patrick Maeght and Martine Lafon greatly contributed to the data archiving. CLIMSO-DB is hosted and taken care of by the OV-GSO virtual observatory (https://ov-gso.irap.omp.eu/). In memory of Laurent Parès, optician engineer at IRAP who contributed greatly to the design of CLIMSO's optics, and Claude Frelat, the diligent and always joyful treasurer of the Observateurs associés association. The editor thanks Yoichiro Hanaoka and an anonymous reviewer for their assistance in evaluating this paper.

\section{References}

Arge CN, Pizzo VJ. 2000. Improvement in the prediction of SW conditions using near-real-time solar magnetic field updates. $J$ Geophys Res 105: 10465-10479.

Burt J, Smith B. 2012. Deep space climate observatory: The DSCOVR mission. In: 2012 IEEE Aerospace Conference, Big Sky, MT, pp. 1-13. https://doi.org/10.1109/AERO.2012.6187025.

Chen P, Ding M, Fang C. 2005. Synthesis of CME-associated moreton and EIT wave features from MHD simulations. Space Sci Rev 121: 201-211. https://doi.org/10.1007/s11214-006-3911-0.

Chen PF. 2011. Coronal mass ejections: Models and their observational basis. Living Rev Sol Phys 8: 1. https://doi.org/10.12942/ lrsp-2011-1.

Cliver EW, Webb DF, Howard RA. 1999. On the origin of solar metric type II bursts. Sol Phys 187: 89.

Demarcq J, Hugon M, Rösch J, Trellis M. 1965. Un coronographe achromatique amélioré pour la spectrographie de la couronne solaire. C.R. Acad Sci Paris 261: 4629.

Dettwiller L, Noëns J-C. 2008. Les nouveaux coronographes du Pic du Midi: Schémas optiques et buts scientifiques. BUP 102: 1227 1248.

Erard S, Cecconi B, Le Sidaner P, Berthier J, Henry F, et al. 2014. The EPN-TAP protocol for the Planetary Science Virtual Observatory. Astron Comput 7-8: 52-61. ISSN 2213-1337. https://doi. org/10.1016/j.ascom.2014.07.008.

Erard S, Cecconi B, Le Sidaner P, Rossi AP, Capria MT, et al. 2018. VESPA: A community-driven Virtual Observatory in Planetary Science. Planet Space Sci 150: 65-85. https://doi.org/10.1016/j. pss.2017.05.013.

Génot V, Budnik E, Jacquey C, Bouchemit M, Renard B, et al. 2020. Automated Multi-Dataset Analysis (AMDA): an on-line database and analysis tool for heliospheric and planetary plasma data. Submitted to Planetary Space Science.

Hill F, Martens P, Yoshimura K, Gurman J, Hourclé J, et al. 2009. The virtual solar observatory - A resource for International Heliophysics Research. Earth Moon Planet 104: 315-330. https://doi.org/10.1007/s11038-008-9274-7.

Jacquey C, Génot V, Budnik H, Hitier R, Bouchemit M, et al. 2010. AMDA, automated multi-dataset analysis: A web-based service provided by the CDPP. In: The Cluster Active Archive. Astrophysics and Space Science Proceedings, Laakso H, Taylor M, Escoubet C (Eds.), Springer, Dordrecht. https://doi.org/10.1007/ 978-90-481-3499-1_16.

Koechlin L, Dettwiller L, Audejean M, Valais M, López Ariste A. 2019. Solar survey at Pic du Midi: Calibrated data and improved images. A\&A 631: A55. https://doi.org/10.1051/0004-6361/ 201732504.

López Ariste A, Facchin M. 2018. Superoscillations in solar MHD waves and their possible role in heating coronal loops. A\&A 615: A145. https://doi.org/10.1051/0004-6361/201731401.

Lyot B. 1930. La couronne solaire étudiée en dehors des éclipses. Bull Astron 6: 305-316.

Lyot B. 1935. Quelques observations de la couronne solaire et des protubérances en 1935. L'Astronomie 51: 203-218.

Marchand E. 1904. La perturbation magnétique du 31 octobre 1903, Bulletin de la Société Astronomique de France et Revue Mensuelle d'Astronomie, de Météorologie et de Physique du. Bulletin de la Société Astronomique de France et Revue Mensuelle d'Astronomie, de Météorologie et de Physique du Globe 18: 34-36. 
Meunier N, Noëns J-C, Romeuf D, Koutchmy S, Jimenez R., Wurmser O., Rochain S., The OA Team. 2007. Coronagraphic Broad-Band $\mathrm{H} \alpha$ Observations 1998 - 2000. In: The Physics of Chromospheric Plasmas, ASP Conference Series, Vol. 368.

Moreton GE. 1960. H $\alpha$ observations of flare-initiated disturbances with velocities $\sim 1000 \mathrm{~km} / \mathrm{sec}$. Astron J 65: 494 .

Noëns J, Wurmser O. 2000. Ground based limb survey of the sun polar regions in the $\mathrm{H} \alpha$ line. Astrophys Space Sci 273: 17-23. https://doi.org/10.1023/A:1002687606153.

Odstrcil D, Pizzo VJ. 1999. Distortion of the interplanetary magnetic field by three-dimensional propagation of coronal mass ejections in a structured solar wind. J Geophys Res 104(A12): 2822528240. https://doi.org/10.1029/1999JA900319.

Oranje BJ. 1983. The CaII K emission from the Sun as a star, I. observational parameters. A\&A 122: 88-94.

Pariat E, Schmieder B, Berlicki A, Deng Y, Mein N, López Ariste A, Wang S. 2007. Spectrophotometric analysis of Ellerman bombs in the Ca II, $\mathrm{H} \alpha$, and UV range. $A \& A$ 473: 279-289.

Pomoell J, Poedts S. 2018. EUHFORIA: European heliospheric forecasting information asset. J Space Weather Space Clim 8: A35. https://doi.org/10.1051/swsc/2018020.

Romeuf D, Meunier N, Noëns J-C, Koutchmy S, Jimenez R, Wurmser O, Rochain S, "Observateurs Associés" Team. 2007. Analysis of broad-band $\mathrm{H} \alpha$ coronagraphic observations. A\&A 462 (2): 731-741. https://doi.org/10.1051/0004-6361:20054159.
Roudier T, Schmieder B, Filippov B, Chandra R, Malherbe J-M. 2018. Photospheric flows trigger a filament eruption. A\&A 618: A43. https://doi.org/10.1051/0004-6361/201832937.

Rouillard AP, Lavraud B, Génot V, Bouchemit M, Dufourg N, et al. 2017. A propagation tool to connect remote-sensing observations with in-situ measurements of heliospheric structures. Planet Space Sci 147: 61-77. https://doi.org/10.1016/j.pss.2017.07.001.

Sanguillon M, Glorian J-M, Vastel C. 2019. The OV-GSO data center. Astron Data Anal Softw Syst XXVIII 523: 449.

Stone EC, Frandsen AM, Mewaldt RA, Christian ER, Margolies D, Ormes JF, Snow F. 1998. The advanced composition explorer. Space Sci Rev 86: 1-22. https://doi.org/10.1023/A:1005082526237.

Taylor MB. 2005. TOPCAT \& STIL: Starlink Table/VOTable Processing Software. Astron Data Anal Softw Syst XIV 347: 9.

Vernazza JE, Avrett EH, Loeser R. 1981. Structure of the solar chromosphere. III. Models of the EUV brightness components of the quiet sun. Astrophys J Suppl Ser 45: 635-725.

Wang H, Liu C, Wang S, Deng N, Xu Y, Jing J, Cao W. 2013. Study of rapid formation of a $\delta$ sunspot associated with the 2012 July 2 C7.4 flare using high-resolution observations of the New Solar Telescope. Astrophys $J$ L24: 774.

Yurchyshyn V, Wang H, Abramenko V. 2003. How directions and helicity of erupted solar magnetic fields define geoeffectiveness of coronal mass ejections. Adv Space Res 32(10): 1965-1970. https://doi.org/10.1016/S0273-1177(03)90634-X.

Cite this article as: Pitout F, Koechlin L, Loepez Ariste A, Dettwiller L \& Glorian J-M, et al. 2020. Solar surveillance with CLIMSO: instrumentation, database and on-going developments. J. Space Weather Space Clim. 10, 47. https://doi.org/10.1051/swsc/2020039 